\title{
Evolutionary Diversification of Primary Metabolism and Its Contribution to Plant Chemical Diversity
}

\author{
Hiroshi A. Maeda* \\ Department of Botany, University of Wisconsin-Madison, Madison, WI, United States
}

\section{OPEN ACCESS}

Edited by:

Kazuki Saito,

RIKEN Center for Sustainable Resource Science (CSRS), Japan

Reviewed by:

Anthony Michael,

UT Southwestern Medical Center, United States

Philipp Zerbe,

University of California, Davis,

United States

*Correspondence:

Hiroshi A. Maeda

maeda2@wisc.edu

Specialty section:

This article was submitted to Plant Metabolism

and Chemodiversity,

a section of the journal

Frontiers in Plant Science

Received: 01 May 2019

Accepted: 20 June 2019

Published: 10 July 2019

Citation:

Maeda HA (2019) Evolutionary Diversification of Primary Metabolism and Its Contribution to Plant Chemical

Diversity. Front. Plant Sci. 10:881.

doi: 10.3389/fp/s.2019.00881
Plants produce a diverse array of lineage-specific specialized (secondary) metabolites, which are synthesized from primary metabolites. Plant specialized metabolites play crucial roles in plant adaptation as well as in human nutrition and medicine. Unlike welldocumented diversification of plant specialized metabolic enzymes, primary metabolism that provides essential compounds for cellular homeostasis is under strong selection pressure and generally assumed to be conserved across the plant kingdom. Yet, some alterations in primary metabolic pathways have been reported in plants. The biosynthetic pathways of certain amino acids and lipids have been altered in specific plant lineages. Also, two alternative pathways exist in plants for synthesizing primary precursors of the two major classes of plant specialized metabolites, terpenoids and phenylpropanoids. Such primary metabolic diversities likely underlie major evolutionary changes in plant metabolism and chemical diversity by acting as enabling or associated traits for the evolution of specialized metabolic pathways.

\footnotetext{
Keywords: plant chemical diversity, metabolic enzymes, primary metabolism, specialized metabolism, evolution of plant metabolism, amino acid biosynthesis
}

\section{INTRODUCTION}

Plants produce a diverse array of secondary or specialized metabolites, which play critical roles in plant adaptation under various environmental conditions. These phytochemicals are also widely used in human nutrition and medicine. Nearly one million metabolites are estimated to be produced throughout the plant kingdom (Afendi et al., 2012), though many of them are yet to be discovered. All of these specialized metabolites are synthesized from a certain primary metabolite precursor(s), such as sugars, amino acids, nucleotides, organic acids, and fatty acids, which are essential for maintaining cellular homeostasis and the life of whole organisms. Besides their vital nature, primary metabolic pathways are highly regulated and integrated to complex metabolic networks (Baghalian et al., 2014; Sulpice and McKeown, 2015; Beckers et al., 2016; Filho et al., 2018). Consequently, genes encoding primary metabolic enzymes are subjected to purifying selection and generally considered to be conserved among the plant kingdom, unlike highly diversified 
specialized metabolism (Pichersky and Lewinsohn, 2011; Weng et al., 2012; Moghe and Last, 2015; Moore et al., 2019). Yet, some primary metabolic pathways were altered during plant evolution, which had profound impacts on overall plant physiology, metabolism, and adaptation. This review describes examples of primary metabolic diversification in different plant lineages and discusses their potential roles in the evolution of downstream specialized metabolic pathways and plant chemical diversity as enabling or associated traits.

\section{ENABLERS OF EVOLUTIONARY DIVERSIFICATION OF THE PHOTOSYNTHETIC CARBON FIXATION PATHWAYS}

One of the most fundamental metabolic pathways of plants, photosynthetic carbon fixation, has been modified in a number of plant lineages to what is known as $\mathrm{C}_{4}$ photosynthesis and Crassulacean acid metabolism, though the former will be mainly discussed here. Unlike 3-phosphoglycerate (3PGA), a three carbon molecule produced by ribulose-1,5-bisphosphate carboxylase/oxygenase (Rubisco) in $\mathrm{C}_{3}$ photosynthesis, $\mathrm{C}_{4}$ photosynthesis initially generates a four carbon molecule, i.e., oxaloacetate, by phosphoenolpyruvate (PEP) carboxylase (PEPC). Oxaloacetate is further converted to malate or aspartate and shuttled from mesophyll to bundle sheath cells, where $\mathrm{CO}_{2}$ is released for refixation by Rubisco (Figure 1) (Langdale, 2011; Sage et al., 2012; Furbank, 2016). This highly intricate mechanism is seemingly maladaptive due to high metabolic costs (e.g., fixing carbon twice, regeneration of PEP), but provides adaptive advantage under arid, warm, and high light conditions by concentrating $\mathrm{CO}_{2}$ and attenuating the oxygenation side reaction of Rubisco and hence photorespiration (Christin and Osborne, 2014; Sage and Stata, 2015). Thus, besides the decline in atmospheric $\mathrm{CO}_{2}$ around 30 million years ago (Pagani et al., 2005), such extreme environmental conditions, in which some plants existed, likely acted as an "environmental enabler" for the evolutionary diversification of the photosynthetic carbon fixation, the entry step of plant metabolic pathways.

The $\mathrm{C}_{4}$ photosynthetic pathway evolved more than 60 times independently across the plant phylogeny (Sage et al., 2011, 2012). Notably, $C_{4}$ photosynthesis is unevenly distributed across the phylogeny and particularly prevalent in specific plant lineages, such as Poaceae and Caryophyllales (Christin et al., 2009, 2015; Sage et al., 2011). Recent comparative analyses of $\mathrm{C}_{3}$ and $\mathrm{C}_{4}$ plants as well as $\mathrm{C}_{3}-\mathrm{C}_{4}$ transitory species revealed that the repeated evolution of $\mathrm{C}_{4}$ photosynthesis was likely facilitated by certain "pre-conditions" or "enabling traits" that emerged or were present in certain plant lineages (Ludwig, 2013; Sage et al., 2014; Heckmann, 2016; Miyake, 2016; Schlüter and Weber, 2016). These enabling traits include "genetic enablers," such as $\mathrm{C}_{4}$-like cell-type specific expression of $\mathrm{C}_{4}$ enzymes (e.g., PEPC, Williams et al., 2012; Christin et al., 2013a, 2015) and "anatomical enablers," such as protoKranz anatomy (Christin et al., 2013b; Lundgren et al., 2014;
Sage et al., 2014), in $C_{3}$ ancestors. These pre-conditions further facilitated emergence of "metabolic enablers," such as shuttling of photorespiratory glycine from mesophyll to bundle sheath cells acting as $\mathrm{CO}_{2}$ pump (Sage et al., 2013; Schulze et al., 2013). This so-called $C_{2}$ photosynthesis is present in many sister species to $\mathrm{C}_{4}$ lineages (Sage et al., 2011, 2012; Khoshravesh et al., 2016) and appears to be accompanied by shuttling of other metabolites, such as alanine/pyruvate or aspartate/malate, for balancing of nitrogen between the mesophyll and bundle sheath cells (Mallmann et al., 2014; Schlüter and Weber, 2016). Once these pre-conditions were established, $\mathrm{C}_{4}$ photosynthesis could evolve relatively easily and thus repeatedly, such as through optimization of kinetic properties of $\mathrm{C}_{4}$ enzymes (e.g., PEPC) and bundle sheath specific expression of Rubisco (Langdale, 2011; Sage et al., 2012; Furbank, 2016; Reeves et al., 2017). Thus, the combination of environmental, genetic, anatomical, and metabolic enablers allowed astounding alterations in the core primary metabolic pathway, photosynthetic carbon fixation, in certain plant lineages.

\section{DIVERSIFICATION OF AMINO ACID BIOSYNTHETIC PATHWAYS AT THE INTERFACE OF PRIMARY AND SPECIALIZED METABOLIC PATHWAYS}

Amino acid biosynthetic pathways not only provide essential protein building blocks but connect central carbon metabolism to a variety of specialized metabolism. Some of these amino acid pathways have diversified in certain plant lineages and likely contributed to the chemical diversity of their downstream specialized metabolism.

Isopropylmalate synthase (IPMS) catalyzes the committed step of leucine biosynthesis (de Kraker et al., 2007). IPMS competes for the 3-methyl-2-oxobutanoate (3MOB) substrate with valine biosynthesis (Figure 1) and is typically feedback inhibited by the end product, leucine, through its C-terminal allosteric regulatory domain (Koon et al., 2004; de Kraker and Gershenzon, 2011). Glandular trichomes of Solanaceae plants accumulate insecticidal specialized metabolites, acylsugars, which have various aliphatic acids attached to a sugar backbone (e.g., sucrose, Fan et al., 2019). A wild tomato Solanum pennellii and the cultivated tomato, Solanum lycopersicum, have 2-methylpropanoic and 3-methylbutanoic acid (iC4 and iC5) acyl chains, which are derived from $3 \mathrm{MOB}$ and 3isopropylmalate, intermediates of valine and leucine metabolism, respectively (Figure 1). Analysis of introgression lines between S. lycopersicum and $S$. pennellii, followed by expression and biochemical analyses, revealed that the C-terminal regulatory domain of the IPMS3 isoform is truncated in S. lycopersicum, making this isoform insensitive to leucinemediated feedback inhibition (Schilmiller et al., 2010; Ning et al., 2015). In contrast, the IPMS3 isoform of S. pennellii is further truncated into its catalytic domain and has lost the enzyme activity. Thus, the de-regulated and inactive IPMS3 in S. lycopersicum and S. pennellii directs more carbon flow 


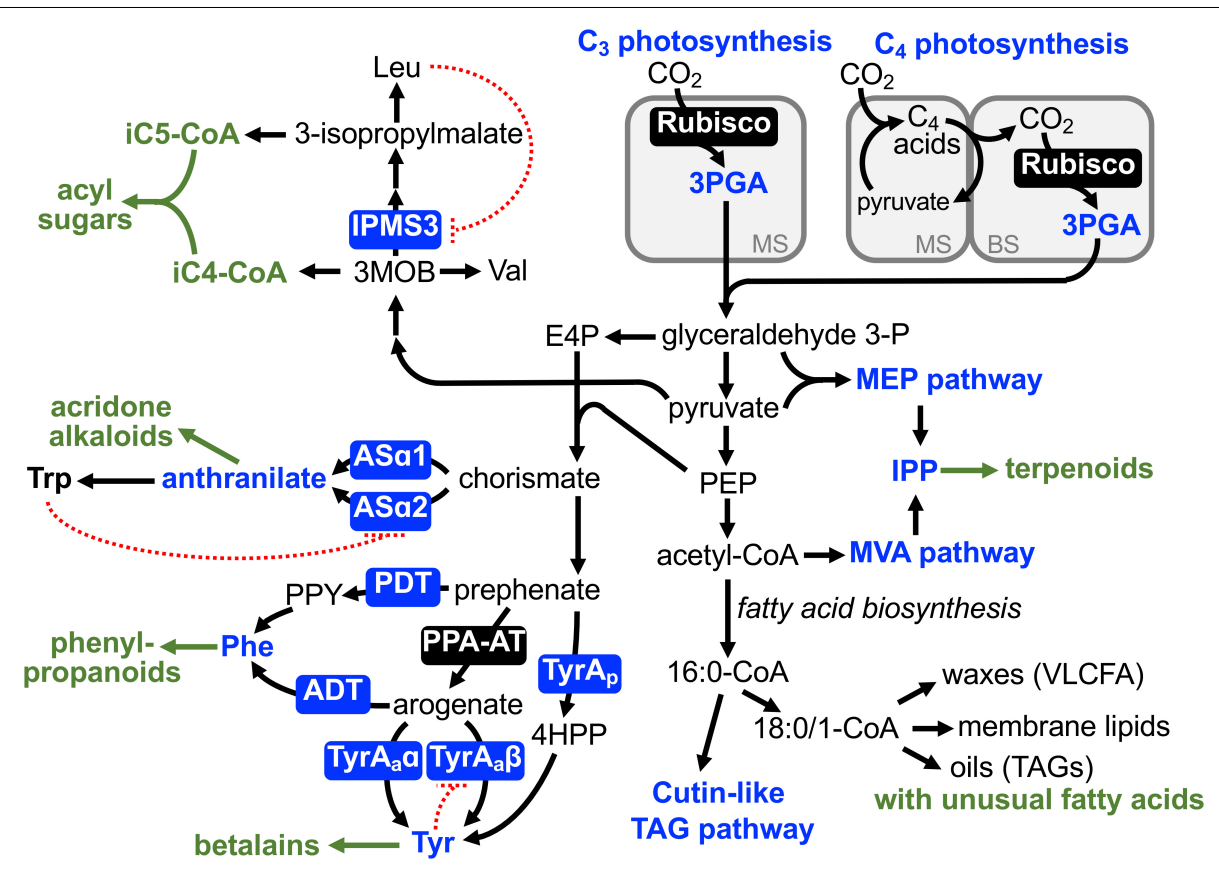

FIGURE 1 | Diversification of primary metabolic pathways and enzymes in plants. Although primary metabolism is highly constrained and generally assumed to be conserved within the plant kingdom, there are examples of evolutionary diversification of some primary metabolic pathways (blue). Many of them likely supported diversification of downstream specialized metabolism (green letters) as enabling or associated traits. Blue letters and boxes denote alternative routes or enzymes to synthesize key primary metabolite precursors. Green arrows and letters indicate specialized metabolic pathways derived from these primary precursors. Dotted red lines indicate feedback inhibition that act specifically on canonical leucine (Leu), tryptophan (Trp), and tyrosine (Tyr) biosynthetic pathways, but not on "lineage-specific" alternative enzymes. ADT, arogenate dehydratase; ASa, anthranilate synthase a subunit; BS, bundle sheath cells; E4P, erythrose 4-phosphate; 4HPP, 4-hydroxyphenylpyruvate; iC4-CoA, 2-methylpropanoic-coenzyme A; iC5-CoA, 2-methylbutanoic-coenzyme A; IPMS, isopropylmalate synthase; IPP, isopentenyl diphosphate; MEP, methylerythritol phosphate; 3MOB, 3-methyl-2-oxobutanoate; MS, mesophyll cells; MVA, mevalonate; PDT, prephenate dehydratase; PEP, phosphoeno/pyruvate; 3PGA, 3-phosphoglycerate; PPA-AT, prephenate aminotransferase; PPY, phenylpyruvate; TAG, triacylglycerol; TyrAa, arogenate dehydrogenase; TyrAp, prephenate dehydrogenase; Val, valine; VLCFA, very long chain fatty acid.

toward leucine and valine metabolism, respectively. Having the broad substrate specificity of downstream acyl-CoAdependent acyltransferase (Schilmiller et al., 2015), increased availability of $3 \mathrm{MOB}$ and 3-isopropylmalate contributes to the formation of $\mathrm{iC} 4$ and iC5 acylsugars, respectively. Brassicaceae species including Arabidopsis thaliana also has a truncated IPMS homolog but with point mutations that alter substrate specificity to now function as methylthioalkylmalate synthase in the initial step of methionine-derived glucosinolate biosynthesis (de Kraker and Gershenzon, 2011). Unlike the latter example of recruitment of specialized metabolic enzymes from primary metabolism, as discussed in previous reviews (Weng, 2014; Moghe and Last, 2015), the study by Ning et al. (2015) revealed a role of altered branch chain amino acid biosynthesis in the acyl chain diversity of acylsugars in the Solanum genus.

Anthranilate synthase (AS) catalyzes the committed step of biosynthesis of an aromatic amino acid, L-tryptophan, and its enzyme activity is strictly regulated through feedback inhibition of one of the AS enzyme complex, ASa, by tryptophan (Romero et al., 1995; Li and Last, 1996). Two copies of ASa genes, ASa 1 and ASa2, were found in Ruta graveolens (the Rutaceae family) that uses anthranilate to produce unique specialized metabolites, acridone alkaloids (Bohlmann et al., 1995). While
ASa2 was constitutively expressed, ASa1 was induced under elicitor treatment, which stimulates the accumulation of acridone alkaloids. Interestingly, the ASa1 enzyme was much more resistant than $A S a 2$ to the tryptophan-mediated feedback inhibition, suggesting that the expression of the de-regulated ASal enzyme allowed elevated accumulation of the anthranilate precursor and hence efficient production of the downstream specialized metabolites, acridone alkaloids, in this unique plant lineage (Bohlmann et al., 1996; Figure 1). A naturally occurring feedback-insensitive ASa enzyme has also been identified in Nicotiana tabacum (the Solanaceae family, Song et al., 1998), but its in planta function is currently unknown. Further evolutionary analyses across the Rutaceae family can evaluate if the increased availability of anthranilate served as an enabling trait for later evolution of acridone alkaloid biosynthesis. Alternatively, the de-regulated ASa1 might have evolved after the emergence of the acridone alkaloid pathway as an associated trait and further elevated the alkaloid production.

L-Tyrosine is another aromatic amino acid required for protein synthesis but also used to produce diverse plant natural products, such as tocochromanols, benzylisoquinoline alkaloids, cyanogenic glycosides (e.g., dhurrin), and rosmarinic acids (Schenck and Maeda, 2018). Tyrosine is typically produced 
via arogenate dehydrogenase $\left(\operatorname{TyrA}_{a}\right)$ that is localized within the plastids (Rippert et al., 2009; Wang et al., 2016) and strongly feedback inhibited by tyrosine (Figure 1; Connelly and Conn, 1986; Rippert and Matringe, 2002a,b). Recent studies, however, uncovered diversification of the tyrosine biosynthetic pathways in different plant lineages. In addition to the highly regulated plastidic $\mathrm{TyrA}_{\mathrm{a}}$-mediated pathway, many legumes including Glycine max (soybean) and Medicago truncatula have an additional tyrosine biosynthetic pathway mediated by prephenate dehydrogenase $\left(\operatorname{TyrA}_{\mathrm{p}}\right)$ (Rubin and Jensen, 1979; Schenck et al., 2015), which is often found in microbes (Bonner and Jensen, 1987; Bonner et al., 2008; Schenck et al., 2017b). Notably, these legume TyrA $A_{p}$ enzymes are localized outside of the plastids and completely insensitive to feedback inhibition by tyrosine (Schenck et al., 2015, 2017a), suggesting that the alternative tyrosine pathway is physically separated from the canonical plastidic pathway and escaped feedback inhibition by tyrosine (Figure 1). While the metabolic and physiological functions of the alternative cytosolic $\operatorname{TyrA}_{\mathrm{p}}$ pathway in legumes is largely unknown, some legumes accumulate very high levels of tyrosine and tyrosine-derived compounds (e.g., L-DOPA in Mucuna pruriens, Wichers et al., 1993; Lokvam et al., 2006). A recent study found that the expression of gene encoding the tyrosine-insensitive $\operatorname{TyrA}_{\mathrm{p}}$ enzyme is elevated in Inga species that accumulate tyrosine and its derived secondary metabolites (e.g., tyrosine-gallates) at 5 to $20 \%$ of seedling dry weight (Coley et al., 2019). Thus, the presence of the feedback-insensitive TyrA $\mathrm{A}_{\mathrm{p}}$ enzyme in the legume family likely provided a unique pre-condition that enabled increased tyrosine biosynthetic activity and hyperaccumulation of tyrosine-derived compounds in this specific genus of legumes.

Betalains are red to yellow alkaloid pigments uniquely produced in the plant order Caryophyllales, which include Beta vulgaris (beet), spinach, quinoa, and cactus. Betalain pigments are derived from tyrosine and replaced more ubiquitous red to purple anthocyanin pigments derived from phenylalanine in many Caryophyllales species (Tanaka et al., 2008; Brockington et al., 2011; Polturak and Aharoni, 2018; Figure 1). Like Arabidopsis and unlike legumes, Caryophyllales species only have arogenate-specific TyrA $\mathrm{a}_{\mathrm{a}}$ enzymes; however, one $\operatorname{TyrA}_{a}$ isoform $\left(\operatorname{TyrA}_{a} a\right)$ exhibits relaxed sensitivity to tyrosine inhibition (Lopez-Nieves et al., 2018; Figure 1). The presence of the de-regulated $\operatorname{TyrA}_{\mathrm{a}} \mathrm{a}$ enzymes positively and negatively correlates with those of betalain and anthocyanin pigmentation, respectively, across Caryophyllales. Evolutionary analyses, by utilizing transcriptome data of over one hundred Caryophyllales species (Brockington et al., 2015), revealed that the de-regulated TyrA $\mathrm{A}_{\mathrm{a}} \mathrm{a}$ enzymes emerged before the evolution of the betalain biosynthetic pathway (Lopez-Nieves et al., 2018). Thus, the enhanced supply of the tyrosine precursor, due to relaxed regulation of the TyrA $\mathrm{A}_{\mathrm{a}}$ enzyme, likely acted as a metabolic enabler for the subsequent evolution of a novel downstream specialized metabolic pathway, betalain biosynthesis, in this specific plant order (Figure 1). Further evolutionary analyses of associated genes and enzymes involved in the betalain pathway and the competing phenylalanine and phenylpropanoid pathways will provide novel insight into how primary and specialized metabolism evolved coordinately in a macroevolutionary scale beyond the levels of species and genera.

\section{ANCIENT DIVERSIFICATION OF IPP AND PHENYLALANINE BIOSYNTHETIC PATHWAYS IN PLANTAE}

In the ancient history of Plantae, alternative primary metabolic pathways evolved and likely contributed to later evolution of plant specialized metabolism and chemical diversity. Terpenoids and phenylpropanoids are the two major classes of plant natural products, which are synthesized from the primary metabolite precursors, isopentenyl pyrophosphate (IPP) and phenylalanine, respectively (McGarvey and Croteau, 1995; Gershenzon and Dudareva, 2007; Vogt, 2010; Tohge et al., 2013). Notably, plants possess two alternative pathways to synthesize IPP and phenylalanine.

In addition to sterols and quinones, plants use IPP to synthesize photosynthetic pigments (chlorophylls, carotenoids), plant hormones (brassinosteroids, abscisic acid, gibberellins), and a diverse array of terpenoid compounds (McGarvey and Croteau, 1995; Gershenzon and Dudareva, 2007; Tholl, 2015). Such a high demand of IPP for synthesis of diverse terpenoid compounds in plants is supported by the two alternative IPP biosynthetic pathways, the methylerythritol phosphate (MEP) and mevalonate (MVA) pathways, which take place in the plastidic and extra-plastidic subcellular compartments, respectively (Vranová et al., 2013; Rodríguez-Concepción and Boronat, 2015). The MEP pathway utilizes glyceraldehyde 3phosphate derived from the pentose phosphate pathways in the plastids and hence can draw carbon flux directly from photosynthetic carbon fixation (Figure 1). While the MVA pathway appears to be an ancestral pathway that evolved in all three domains of life (i.e., eukaryotes, archaea, and most bacteria) or in their last universal ancestor (i.e., cenancestor) (Lombard and Moreira, 2011), the plastidic MEP pathway has mosaic evolutionary origins (Lange et al., 2000; Matsuzaki et al., 2008). A common ancestor of plastid bearing eukaryotes likely acquired MEP pathway enzymes from various bacterial ancestors (i.e., cyanobacteria, a-proteobacteria, Chlamydia) through horizontal gene transfers (Matsuzaki et al., 2008) and the MEP pathway was vertically transmitted to the descendants, the entire Plantae including algae and plants.

L-Phenylalanine is the primary metabolite precursor of phenylpropanoids and is synthesized via two alternative pathways in plants (Tzin and Galili, 2010; Maeda and Dudareva, 2012; Yoo et al., 2013; Qian et al., 2019). In many microbes, phenylalanine is synthesized via the phenylpyruvate intermediate, catalyzed by prephenate dehydratase (PDT) and phenylpyruvate aminotransferase (Figure 1) (Bentley, 1990). Although an analogous phenylpyruvate pathway also exists in the plant cytosol (Yoo et al., 2013; Qian et al., 2019), plants synthesize phenylalanine mainly in the plastids via the 
L-arogenate intermediate: prephenate is first transaminated by prephenate aminotransferase (PPA-AT) to arogenate (Graindorge et al., 2010; Dal Cin et al., 2011; Maeda et al., 2011), which is then converted to phenylalanine by arogenate dehydratase (ADT; Siehl and Conn, 1988; Cho et al., 2007; Maeda et al., 2010; Figure 1). Evolutionary analyses of the PPA-AT and ADT enzymes suggested that an ancestor of green algae and land plants appear to have acquired both of these two enzymes from an ancestor of Chlorobi/Bacteroidetes bacteria, likely through horizontal gene transfer (Dornfeld et al., 2014). Some cyanobacteria also have PPA-AT enzymes but with a distinct evolutionary origin from those of plants and Chlorobi/Bacteroidetes bacteria (Graindorge et al., 2014; Giustini et al., 2019). Thus, these dual primary metabolic pathways of isoprenoid and phenylalanine biosynthesis appear to have evolved in a common ancestor of Plantae. Although evolutionary analyses of such deep phylogenetic nodes are challenging, these dual precursor supply pathways potentially served as metabolic enablers for the evolutionary expansion of terpenoids and phenylpropanoids, the hallmarks of chemical diversity uniquely seen in the plant kingdom today.

\section{DIVERSIFICATION OF LIPID METABOLISM IN PLANTS}

Notable chemical diversity also exists in plant lipid metabolism (Badami and Patil, 1980; Ohlrogge et al., 2018), which makes the boundary of primary and specialized (secondary) metabolism difficult to define. Besides major acyl chains (e.g., oleic 18:1, linolenic 18:3) found in most plant lipids, some plants produce unusual fatty acids: For example, oils of castor (Ricinus communis, Euphorbiaceae family) and Vernonia galamensis (Asteraceae family) consist of primarily (80-90\%) hydroxylated and epoxy fatty acids, respectively (Canvin, 1963; Ayorinde et al., 1990). Also, diverse acetylenic natural products having a carbon-carbon triple bond(s) or alkynyl functional group can be produced by modification of the fatty acid precursors (Minto and Blacklock, 2008; Negri, 2015). The production of these hydroxylated fatty acids and polyacetylenes are mediated by divergent fatty acid desaturases with altered product specificities and catalytic properties (van de Loo et al., 1995; Broun et al., 1998; Liu et al., 1998; Broadwater et al., 2002; Minto and Blacklock, 2008; Negri, 2015). Tremendous diversity of cuticular waxes has been also documented across the plant kingdom likely due to the presence of specialized acyl chain elongation and modifying enzymes (Jetter et al., 2007; Busta and Jetter, 2018).

Recent studies also revealed an intriguing alteration in the core lipid metabolic pathway, triacylglycerol (TAG) biosynthesis, in a specific plant lineage. The fruits of Bayberry (Myrica pensylvanica, Myricaceae family) accumulate abundant and unusual extracellular glycerolipids: TAG, diacylglycerol (DAG), and monoacylglycerol with completely saturated acyl chains at up to $30 \%$ of fruit dry weight (Harlow et al., 1965; Simpson and Ohlrogge, 2016). This unique surface wax attracts birds for seed dispersal and is used for making scented candles (Fordham, 1983). Fleshy fruits of oil palm, olive, and avocado also accumulate a large quantity of glycerolipids but intracellularly and by upregulating conventional fatty acid and TAG biosynthetic pathways (Bourgis et al., 2011; Kilaru et al., 2015). In contrast, a novel TAG biosynthetic pathway evolved in Bayberry through "re-purposing" genes and enzymes involved in cutin biosynthesis by altering their gene expression (Simpson and Ohlrogge, 2016; Simpson et al., 2016). These alterations include elevated expression of genes encoding the $G$ subfamily of ABC (ABCG) transporters and lipid transporter proteins likely required for lipid transport across cell membranes and walls, respectively, which will allow extracellular formation of TAG (Simpson et al., 2016). It will be interesting to examine how such reprograming of existing lipid metabolic pathways occur in a step-wise manner during evolution, which will provide useful information for engineering other plants to produce and secrete abundant extracellular glycerolipids.

\section{SUMMARY AND PERSPECTIVE}

Although not as frequent as those of specialized metabolism, accumulating evidence indicates that pathways and enzymes of primary metabolism can be diversified during the plant evolution. Such relatively rare alterations in primary metabolism likely contributed to major evolutionary innovations in the plant kingdom, including the evolution of downstream specialized metabolic pathways and hence plant chemical diversity. Some alterations in primary metabolism appear to have acted as enabling traits for the evolution of novel specialized metabolism, at least in the case of de-regulated tyrosine biosynthesis in Caryophyllales that preceded the emergence of betalain pigmentation (Lopez-Nieves et al., 2018). In other instances, primary metabolic alterations likely co-evolved with and support efficient operation of specialized metabolic pathways. It remains to be examined how prevalent the phenomenon is beyond the pathways and plant lineages that have been examined so far and what impacts such primary metabolic diversification had on overall metabolism, physiology, and environmental adaption of diverse plant species. Another intriguing question is how seemingly maladaptive alterations in highly conserved and constrained primary metabolism were maintained in certain plant lineages, especially until the emergence of a new downstream pathway which might have eventually provided adaptive advantage. What are the environmental, anatomical, and genetic enablers underlying primary metabolic diversification? In the case of tomato feedback-insensitive IPMS and legume TyrA enzymes, their specific expression in the apical trichome cells (Ning et al., 2015) and extra-plastidic subcellular compartment (Schenck et al., 2015) likely allow minimal disturbance to de novo biosynthesis of branch chain and aromatic amino acids, respectively. Further addressing these questions will lead to broader understanding of the evolution of plant metabolism at a macroevolutionary scale. The acquired knowledge of primary metabolic diversification and its underlying genetic and biochemical basis will also allow us to redesign plant metabolism in a holistic manner from primary to specialized metabolism. 


\section{AUTHOR CONTRIBUTIONS}

HM wrote the manuscript.

\section{FUNDING}

This work was supported by the National Science Foundation grant IOS-1354971 and the Agriculture and Food Research

\section{REFERENCES}

Afendi, F. M., Okada, T., Yamazaki, M., Hirai-Morita, A., Nakamura, Y., Nakamura, K., et al. (2012). KNApSAcK family databases: integrated metabolite-plant species databases for multifaceted plant research. Plant Cell Physiol. 53:e1. doi: 10.1093/pcp/pcr165

Ayorinde, F. O., Butler, B. D., and Clayton, M. T. (1990). Vernonia galamensis: a rich source of epoxy acid. J. Am. Oil Chem. Soc. 67, 844-845. doi: 10.1007/ BF02540503

Badami, R. C., and Patil, K. B. (1980). Structure and occurrence of unusual fatty acids in minor seed oils. Prog. Lipid Res. 19, 119-153. doi: 10.1016/01637827(80)90002-8

Baghalian, K., Hajirezaei, M. R., and Schreiber, F. (2014). Plant metabolic modeling: achieving new insight into metabolism and metabolic engineering. Plant Cell 26, 3847-3866. doi: 10.1105/tpc.114.130328

Beckers, V., Dersch, L. M., Lotz, K., Melzer, G., Bläsing, O. E., Fuchs, R., et al. (2016). In silico metabolic network analysis of Arabidopsis leaves. BMC Syst. Biol. 10:102. doi: 10.1186/s12918-016-0347-3

Bentley, R. (1990). The shikimate pathway - A metabolic tree with many branches. Crit. Rev. Biochem. Mol. Biol. 25, 307-384. doi: 10.3109/10409239009090615

Bohlmann, J., DeLuca, V., Eilert, U., and Martin, W. (1995). Purification and cDNA cloning of anthranilate synthase from Ruta graveolens: modes of expression and properties of native and recombinant enzymes. Plant J. 7, 491-501. doi: 10.1046/j.1365-313x.1995.7030491.x

Bohlmann, J., Lins, T., Martin, W., and Eilert, U. (1996). Anthranilate synthase from Ruta graveolens. Duplicated AS alpha genes encode tryptophan-sensitive and tryptophan-insensitive isoenzymes specific to amino acid and alkaloid biosynthesis. Plant Physiol. 111, 507-514. doi: 10.1104/pp.111.2.507

Bonner, C., and Jensen, R. (1987). Arogenate dehydrogenase. Methods Enzymol. 142, 488-494. doi: 10.1016/s0076-6879(87)42060-0

Bonner, C. A., Disz, T., Hwang, K., Song, J., Vonstein, V., Overbeek, R., et al. (2008). Cohesion group approach for evolutionary analysis of TyrA, a protein family with wide-ranging substrate specificities. Microbiol. Mol. Biol. Rev 72, 13-53. doi: 10.1128/MMBR.00026-27

Bourgis, F., Kilaru, A., Cao, X., Ngando-Ebongue, G. F., Drira, N., Ohlrogge, J. B., et al. (2011). Comparative transcriptome and metabolite analysis of oil palm and date palm mesocarp that differ dramatically in carbon partitioning. Proc. Natl. Acad. Sci. U.S.A. 108, 12527-12532. doi: 10.1073/pnas.110650 2108

Broadwater, J. A., Whittle, E., and Shanklin, J. (2002). Desaturation and hydroxylation. Residues 148 and 324 of Arabidopsis FAD2, in addition to substrate chain length, exert a major influence in partitioning of catalytic specificity. J. Biol. Chem. 277, 15613-15620. doi: 10.1074/jbc.M200231200

Brockington, S. F., Walker, R. H., Glover, B. J., Soltis, P. S., and Soltis, D. E. (2011). Complex pigment evolution in the Caryophyllales. New Phytol. 190, 854-864. doi: $10.1111 / j .1469-8137.2011 .03687 . x$

Brockington, S. F., Yang, Y., Gandia-Herrero, F., Covshoff, S., Hibberd, J. M., Sage, R. F., et al. (2015). Lineage-specific gene radiations underlie the evolution of novel betalain pigmentation in Caryophyllales. New Phytol. 207, 1170-1180. doi: $10.1111 /$ nph.13441

Broun, P., Shanklin, J., Whittle, E., and Somerville, C. (1998). Catalytic plasticity of fatty acid modification enzymes underlying chemical diversity of plant lipids. Science 282, 1315-1317. doi: 10.1126/science.282.5392.1315

Busta, L., and Jetter, R. (2018). Moving beyond the ubiquitous: the diversity and biosynthesis of specialty compounds in plant cuticular waxes. Phytochem. Rev. 17, 1275-1304. doi: 10.1007/s11101-017-9542-9540
Initiative Competitive grant (2015-67013-22955) from the USDA National Institute of Food and Agriculture to HM.

\section{ACKNOWLEDGMENTS}

I would like to thank Dr. Luke Busta for helpful discussion and suggestions.

Canvin, D. (1963). Formation of oil in seed of Ricinus communis L. Can. J. Biochem. Physiol. 41, 1879-1885. doi: 10.1139/y63-214

Cho, M. H., Corea, O. R. A., Yang, H., Bedgar, D. L., Laskar, D. D., Anterola, A. M., et al. (2007). Phenylalanine biosynthesis in Arabidopsis thaliana Identification and characterization of arogenate dehydratases. J. Biol. Chem. 282, 30827-30835. doi: 10.1074/jbc.m702662200

Christin, P. A., Arakaki, M., Osborne, C. P., and Edwards, E. J. (2015). Genetic enablers underlying the clustered evolutionary origins of $\mathrm{C} 4$ photosynthesis in angiosperms. Mol. Biol. Evol. 32, 846-858. doi: 10.1093/molbev/msu410

Christin, P.-A., Boxall, S. F., Gregory, R., Edwards, E. J., Hartwell, J., and Osborne, C. P. (2013a). Parallel recruitment of multiple genes into C4 photosynthesis. Genome Biol. Evol. 5, 2174-2187. doi: 10.1093/gbe/evt168

Christin, P.-A., Osborne, C. P., Chatelet, D. S., Columbus, J. T., Besnard, G., Hodkinson, T. R., et al. (2013b). Anatomical enablers and the evolution of C4 photosynthesis in grasses. Proc. Natl. Acad. Sci. U.S.A. 110, 1381-1386. doi: $10.1073 /$ pnas. 1216777110

Christin, P.-A., and Osborne, C. P. (2014). The evolutionary ecology of C4 plants. New Phytol. 204, 765-781. doi: 10.1111/nph.13033

Christin, P.-A., Salamin, N., Kellogg, E. A., Vicentini, A., and Besnard, G. (2009). Integrating phylogeny into studies of $\mathrm{C} 4$ variation in the grasses. Plant Physiol. 149, 82-87. doi: 10.1104/pp.108.128553

Coley, P. D., Endara, M.-J., Ghabash, G., Kidner, C. A., Nicholls, J. A., Pennington, R. T., et al. (2019). Macroevolutionary patterns in overexpression of tyrosine: an anti-herbivore defence in a speciose tropical tree genus, Inga (Fabaceae). J. Ecol. 107, 1620-1632. doi: 10.1111/1365-2745.13208

Connelly, J. A., and Conn, E. E. (1986). Tyrosine biosynthesis in Sorghum bicolor: isolation and regulatory properties of arogenate dehydrogenase. Z. Naturforsch. C 41, 69-78. doi: 10.1515/znc-1986-1-212

Dal Cin, V., Tieman, D. M., Tohge, T., McQuinn, R., de Vos, R. C. H., Osorio, S., et al. (2011). Identification of genes in the phenylalanine metabolic pathway by ectopic expression of a MYB transcription factor in tomato Fruit. Plant Cell 23, 2738-2753. doi: 10.1105/tpc.111.086975

de Kraker, J.-W., and Gershenzon, J. (2011). From amino acid to glucosinolate biosynthesis: protein sequence changes in the evolution of methylthioalkylmalate synthase in Arabidopsis. Plant Cell 23, 38-53. doi: 10.1105/tpc.110.079269

de Kraker, J.-W., Luck, K., Textor, S., Tokuhisa, J. G., and Gershenzon, J. (2007). Two Arabidopsis genes (IPMS1 and IPMS2) encode isopropylmalate synthase, the branchpoint step in the biosynthesis of leucine. Plant Physiol. 143, 970-986. doi: 10.1104/pp.106.085555

Dornfeld, C., Weisberg, A. J., Ritesh, K. C., Dudareva, N., Jelesko, J. G., and Maeda, H. A. (2014). Phylobiochemical characterization of classIb aspartate/prephenate aminotransferases reveals evolution of the plant arogenate phenylalanine pathway. Plant Cell 26, 3101-3114. doi: 10.1105/tpc. 114.127407

Fan, P., Leong, B. J., and Last, R. L. (2019). Tip of the trichome: evolution of acylsugar metabolic diversity in Solanaceae. Curr. Opin. Plant Biol. 49, 8-16. doi: 10.1016/j.pbi.2019.03.005

Filho, H. A., Machicao, J., and Bruno, O. M. (2018). A hierarchical model of metabolic machinery based on the kcore decomposition of plant metabolic networks. PLoS One 13:e0195843. doi: 10.1371/journal.pone.019 5843

Fordham, A. J. (1983). Of Birds and Bayberries: seed dispersal and propagation of three Myrica species. Arnoldia 43, 20-23.

Furbank, R. T. (2016). Walking the C4 pathway: past, present, and future. J. Exp. Bot. 67, 4057-4066. doi: 10.1093/jxb/erw161 
Gershenzon, J., and Dudareva, N. (2007). The function of terpene natural products in the natural world. Nat. Chem. Biol. 3, 408-414. doi: 10.1038/nchembio. 2007.5

Giustini, C., Graindorge, M., Cobessi, D., Crouzy, S., Robin, A., Curien, G., et al. (2019). Tyrosine metabolism: identification of a key residue in the acquisition of prephenate aminotransferase activity by $1 \beta$ aspartate aminotransferase. FEBS J. 286, 2118-2134. doi: 10.1111/febs.14789

Graindorge, M., Giustini, C., Jacomin, A. C., Kraut, A., Curien, G., and Matringe, M. (2010). Identification of a plant gene encoding glutamate/aspartateprephenate aminotransferase: the last homeless enzyme of aromatic amino acids biosynthesis. FEBS Lett. 584, 4357-4360. doi: 10.1016/j.febslet.2010. 09.037

Graindorge, M., Giustini, C., Kraut, A., Moyet, L., Curien, G., and Matringe, M. (2014). Three different classes of aminotransferases evolved prephenate aminotransferase functionality in arogenate-competent microorganisms. J. Biol. Chem. 289, 3198-3208. doi: 10.1074/jbc.M113.486480

Harlow, R. D., Litchfield, C., Fu, H.-C., and Reiser, R. (1965). The triglyceride composition of Myrica carolinensis fruit coat fat (bayberry tallow). J. Am. Oil Chem. Soc. 42, 747-750. doi: 10.1007/BF02631853

Heckmann, D. (2016). C4 photosynthesis evolution: the conditional Mt. Fuji. Curr. Opin. Plant Biol. 31, 149-154. doi: 10.1016/j.pbi.2016.04.008

Jetter, R., Kunst, L., and Samuels, A. L. (2007). "Composition of Plant Cuticular Waxes," in Annual Plant Reviews Volume 23: Biology of the Plant Cuticle, eds M. Riederer and C. Muller (Hoboken, NJ: John Wiley \& Sons, Ltd.), 145-181. doi: 10.1002/9781119312994.apr0232

Khoshravesh, R., Stinson, C. R., Stata, M., Busch, F. A., Sage, R. F., Ludwig, M., et al. (2016). C3-C4 intermediacy in grasses: organelle enrichment and distribution, glycine decarboxylase expression, and the rise of $\mathrm{C} 2$ photosynthesis. J. Exp. Bot. 67, 3065-3078. doi: 10.1093/jxb/erw150

Kilaru, A., Cao, X., Dabbs, P. B., Sung, H.-J., Rahman, M. M., Thrower, N., et al. (2015). Oil biosynthesis in a basal angiosperm: transcriptome analysis of Persea americana mesocarp. BMC Plant Biol. 15:203. doi: 10.1186/s12870-015-058 6-582

Koon, N., Squire, C. J., and Baker, E. N. (2004). Crystal structure of LeuA from Mycobacterium tuberculosis, a key enzyme in leucine biosynthesis. Proc. Natl. Acad. Sci. U.S.A. 101, 8295-8300. doi: 10.1073/pnas.0400820101

Langdale, J. A. (2011). C4 cycles: past, present, and future research on C4 Photosynthesis. Plant Cell 23, 3879-3892. doi: 10.1105/tpc.111.092098

Lange, B. M., Rujan, T., Martin, W., and Croteau, R. (2000). Isoprenoid biosynthesis: the evolution of two ancient and distinct pathways across genomes. Proc. Natl. Acad. Sci. U.S.A. 97, 13172-13177. doi: 10.1073/pnas. 240454797

Li, J., and Last, R. L. (1996). The Arabidopsis thaliana trp5 mutant has a feedbackresistant anthranilate synthase and elevated soluble tryptophan. Plant Physiol. 110, 51-59. doi: 10.1104/pp.110.1.51

Liu, L., Hammond, E. G., and Nikolau, B. J. (1998). In vivo studies of the biosynthesis of vernolic acid in the seed of Vernonia galamensis. Lipids 33, 1217-1221. doi: 10.1007/s11745-998-0326-3

Lokvam, J., Brenes-Arguedas, T., Lee, J. S., Coley, P. D., and Kursar, T. A. (2006). Allelochemic function for a primary metabolite: the case of L-tyrosine hyperproduction in Inga umbellifera (Fabaceae). Am. J. Bot. 93, 1109-1115. doi: 10.3732/ajb.93.8.1109

Lombard, J., and Moreira, D. (2011). Origins and early evolution of the mevalonate pathway of isoprenoid biosynthesis in the three domains of life. Mol. Biol. Evol. 28, 87-99. doi: 10.1093/molbev/msq177

Lopez-Nieves, S., Yang, Y., Timoneda, A., Wang, M., Feng, T., Smith, S. A., et al. (2018). Relaxation of tyrosine pathway regulation underlies the evolution of betalain pigmentation in Caryophyllales. New Phytol. 217, 896-908. doi: 10. $1111 /$ nph.14822

Ludwig, M. (2013). Evolution of the C4 photosynthetic pathway: events at the cellular and molecular levels. Photosyn. Res. 117, 147-161. doi: 10.1007/s11120013-9853-y

Lundgren, M. R., Osborne, C. P., and Christin, P. A. (2014). Deconstructing Kranz anatomy to understand C4 evolution. J. Exp. Bot. 65, 3357-3369. doi: 10.1093/ jxb/eru186

Maeda, H., and Dudareva, N. (2012). The shikimate pathway and aromatic amino acid biosynthesis in plants. Annu. Rev. Plant Biol. 63, 73-105. doi: 10.1146/ annurev-arplant-042811-105439
Maeda, H., Shasany, A. K., Schnepp, J., Orlova, I., Taguchi, G., Cooper, B. R., et al. (2010). RNAi suppression of Arogenate Dehydratase1 reveals that phenylalanine is synthesized predominantly via the arogenate pathway in petunia petals. Plant Cell 22, 832-849. doi: 10.1105/tpc.109.073247

Maeda, H., Yoo, H., and Dudareva, N. (2011). Prephenate aminotransferase directs plant phenylalanine biosynthesis via arogenate. Nat. Chem. Biol. 7, 19-21. doi: 10.1038/nchembio.485

Mallmann, J., Heckmann, D., Bräutigam, A., Lercher, M. J., Weber, A. P. M., Westhoff, P., et al. (2014). The role of photorespiration during the evolution of C4 photosynthesis in the genus Flaveria. eLife 3, e02478. doi: 10.7554/eLife. 02478

Matsuzaki, M., Kuroiwa, H., Kuroiwa, T., Kita, K., and Nozaki, H. (2008). A cryptic algal group unveiled: a plastid biosynthesis pathway in the oyster parasite Perkinsus marinus. Mol. Biol. Evol. 25, 1167-1179. doi: 10.1093/ molbev/msn064

McGarvey, D. J., and Croteau, R. (1995). Terpenoid metabolism. Plant Cell 7, 1015-1026. doi: 10.1105/tpc.7.7.1015

Minto, R. E., and Blacklock, B. J. (2008). Biosynthesis and function of polyacetylenes and allied natural products. Prog. Lipid Res. 47, 233-306. doi: 10.1016/j.plipres.2008.02.002

Miyake, H. (2016). Starch accumulation in the bundle sheaths of C3 plants: a possible pre-condition for C4 Photosynthesis. Plant Cell Physiol. 57, 890-896. doi: $10.1093 / \mathrm{pcp} / \mathrm{pcw} 046$

Moghe, G. D., and Last, R. L. (2015). Something old, something new: conserved enzymes and the evolution of novelty in plant specialized metabolism. Plant Physiol. 169, 1512-1523. doi: 10.1104/pp.15.00994

Moore, B. M., Wang, P., Fan, P., Leong, B., Schenck, C. A., Lloyd, J. P., et al. (2019). Robust predictions of specialized metabolism genes through machine learning. Proc. Natl. Acad. Sci. U.S.A. 116, 2344-2353. doi: 10.1073/pnas.1817074116

Negri, R. (2015). Polyacetylenes from terrestrial plants and fungi: recent phytochemical and biological advances. Fitoterapia 106, 92-109. doi: 10.1016/j. fitote.2015.08.011

Ning, J., Moghe, G. D., Leong, B., Kim, J., Ofner, I., Wang, Z., et al. (2015). A feedback-insensitive Isopropylmalate synthase affects acylsugar composition in cultivated and wild tomato. Plant Physiol. 169, 1821-1835. doi: 10.1104/pp.15. 00474

Ohlrogge, J., Thrower, N., Mhaske, V., Stymne, S., Baxter, M., Yang, W., et al. (2018). PlantFAdb: a resource for exploring hundreds of plant fatty acid structures synthesized by thousands of plants and their phylogenetic relationships. Plant J. 96, 1299-1308. doi: 10.1111/tpj.14102

Pagani, M., Zachos, J. C., Freeman, K. H., Tipple, B., and Bohaty, S. (2005). Marked decline in atmospheric carbon dioxide concentrations during the Paleogene. Science 309, 600-603. doi: 10.1126/science.1110063

Pichersky, E., and Lewinsohn, E. (2011). Convergent evolution in plant specialized metabolism. Annu. Rev. Plant Biol. 62, 549-566. doi: 10.1146/annurev-arplant042110-103814

Polturak, G., and Aharoni, A. (2018). "La Vie en Rose": biosynthesis, sources, and applications of betalain pigments. Mol. Plant 11, 7-22. doi: 10.1016/j.molp. 2017.10.008

Qian, Y., Lynch, J. H., Guo, L., Rhodes, D., Morgan, J. A., and Dudareva, N. (2019). Completion of the cytosolic post-chorismate phenylalanine biosynthetic pathway in plants. Nat. Commun. 10:15. doi: 10.1038/s41467-018-07969-7962

Reeves, G., Grangé-Guermente, M. J., and Hibberd, J. M. (2017). Regulatory gateways for cell-specific gene expression in C4 leaves with Kranz anatomy. J. Exp. Bot. 68, 107-116. doi: 10.1093/jxb/erw438

Rippert, P., and Matringe, M. (2002a). Molecular and biochemical characterization of an Arabidopsis thaliana arogenate dehydrogenase with two highly similar and active protein domains. Plant Mol. Biol. 48, 361-368.

Rippert, P., and Matringe, M. (2002b). Purification and kinetic analysis of the two recombinant arogenate dehydrogenase isoforms of Arabidopsis thaliana. Eur. J. Biochem. 269, 4753-4761. doi: 10.1046/j.1432-1033.2002.03 172.x

Rippert, P., Puyaubert, J., Grisollet, D., Derrier, L., and Matringe, M. (2009). Tyrosine and phenylalanine are synthesized within the plastids in Arabidopsis. Plant Physiol. 149, 1251-1260. doi: 10.1104/pp.108.130070

Rodríguez-Concepción, M., and Boronat, A. (2015). Breaking new ground in the regulation of the early steps of plant isoprenoid biosynthesis. Curr. Opin. Plant Biol. 25, 17-22. doi: 10.1016/j.pbi.2015.04.001 
Romero, R. M., Roberts, M. F., and Phillipson, J. D. (1995). Anthranilate synthase in microorganisms and plants. Phytochemistry 39, 263-276. doi: 10.1016/00319422(95)00010-15

Rubin, J. L., and Jensen, R. A. (1979). Enzymology of L-tyrosine biosynthesis in mung bean (Vigna radiata [L.] Wilczek). Plant Physiol. 64, 727-734. doi: 10.1104/pp.64.5.727

Sage, R. F., Christin, P.-A., and Edwards, E. J. (2011). The C4 plant lineages of planet earth. J. Exp. Bot. 62, 3155-3169. doi: 10.1093/jxb/err048

Sage, R. F., Khoshravesh, R., and Sage, T. L. (2014). From proto-Kranz to C4 Kranz: building the bridge to C4 photosynthesis. J. Exp. Bot. 65, 3341-3356. doi: $10.1093 /$ jxb/eru180

Sage, R. F., Sage, T. L., and Kocacinar, F. (2012). Photorespiration and the evolution of C4 photosynthesis. Annu. Rev. Plant Biol. 63, 19-47. doi: 10.1146/annurevarplant-042811-105511

Sage, R. F., and Stata, M. (2015). Photosynthetic diversity meets biodiversity: the C4 plant example. J. Plant Physiol. 172, 104-119. doi: 10.1016/j.jplph.2014.07.024

Sage, T. L., Busch, F. A., Johnson, D. C., Friesen, P. C., Stinson, C. R., Stata, M., et al. (2013). Initial events during the evolution of C4 photosynthesis in 3 species of Flaveria. Plant Physiol. 163, 1266-1276. doi: 10.1104/pp.113.221119

Schenck, C. A., Chen, S., Siehl, D. L., and Maeda, H. A. (2015). Non-plastidic, tyrosine-insensitive prephenate dehydrogenases from legumes. Nat. Chem. Biol. 11, 52-57. doi: 10.1038/nchembio.1693

Schenck, C. A., Holland, C. K., Schneider, M. R., Men, Y., Lee, S. G., Jez, J. M., et al. (2017a). Molecular basis of the evolution of alternative tyrosine biosynthetic routes in plants. Nat. Chem. Biol. 13, 1029-1035. doi: 10.1038/nchembio.2414

Schenck, C. A., Men, Y., and Maeda, H. A. (2017b). Conserved molecular mechanism of TyrA dehydrogenase substrate specificity underlying alternative tyrosine biosynthetic pathways in plants and microbes. Front. Mol. Biosci 4:73. doi: $10.3389 /$ fmolb.2017.00073

Schenck, C. A., and Maeda, H. A. (2018). Tyrosine biosynthesis, metabolism, and catabolism in plants. Phytochemistry 149, 82-102. doi: 10.1016/j.phytochem. 2018.02.003

Schilmiller, A., Shi, F., Kim, J., Charbonneau, A. L., Holmes, D., Daniel Jones, A., et al. (2010). Mass spectrometry screening reveals widespread diversity in trichome specialized metabolites of tomato chromosomal substitution lines. Plant J. 62, 391-403. doi: 10.1111/j.1365-313X.2010.04154.X

Schilmiller, A. L., Moghe, G. D., Fan, P., Ghosh, B., Ning, J., Jones, A. D., et al. (2015). Functionally divergent alleles and duplicated Loci encoding an acyltransferase contribute to acylsugar metabolite diversity in Solanum trichomes. Plant Cell 27, 1002-1017. doi: 10.1105/tpc.15.00087

Schlüter, U., and Weber, A. P. M. (2016). The road to C4 photosynthesis: evolution of a complex trait via intermediary states. Plant Cell Physiol. 57, 881-889. doi: 10.1093/pcp/pcw009

Schulze, S., Mallmann, J., Burscheidt, J., Koczor, M., Streubel, M., Bauwe, H., et al. (2013). Evolution of C4 photosynthesis in the genus Flaveria: establishment of a photorespiratory $\mathrm{CO}_{2}$ pump. Plant Cell 25, 2522-2535. doi: 10.1105/tpc.113. 114520

Siehl, D. L., and Conn, E. E. (1988). Kinetic and regulatory properties of arogenate dehydratase in seedlings of Sorghum bicolor (L.) Moench. Arch. Biohchem. Biophys. 260, 822-829. doi: 10.1016/0003-9861(88)90513-9

Simpson, J. P., and Ohlrogge, J. B. (2016). A novel pathway for triacylglycerol biosynthesis is responsible for the accumulation of massive quantities of glycerolipids in the surface wax of bayberry (Myrica pensylvanica) fruit. Plant Cell 28, 248-264. doi: 10.1105/tpc.15.00900

Simpson, J. P., Thrower, N., and Ohlrogge, J. B. (2016). How did nature engineer the highest surface lipid accumulation among plants? Exceptional expression of acyl-lipid-associated genes for the assembly of extracellular triacylglycerol by
Bayberry (Myrica pensylvanica) fruits. Biochim. Biophys. Acta 1861, 1243-1252. doi: 10.1016/j.bbalip.2016.01.022

Song, H. S., Brotherton, J. E., Gonzales, R. A., and Widholm, J. M. (1998). Tissue culture-specific expression of a naturally occurring tobacco feedbackinsensitive anthranilate synthase. Plant Physiol. 117, 533-543. doi: 10.1104/pp. 117.2.533

Sulpice, R., and McKeown, P. C. (2015). Moving toward a comprehensive map of central plant metabolism. Annu. Rev. Plant Biol. 66, 187-210. doi: 10.1146/ annurev-arplant-043014-114720

Tanaka, Y., Sasaki, N., and Ohmiya, A. (2008). Biosynthesis of plant pigments: anthocyanins, betalains and carotenoids. Plant J. 54, 733-749. doi: 10.1111/j. 1365-313X.2008.03447.x

Tholl, D. (2015). Biosynthesis and biological functions of terpenoids in plants. $A d v$. Biochem. Eng. Biotechnol. 148, 63-106. doi: 10.1007/10_2014_295

Tohge, T., Watanabe, M., Hoefgen, R., and Fernie, A. R. (2013). The evolution of phenylpropanoid metabolism in the green lineage. Crit. Rev. Biochem. Mol. Biol. 48, 123-152. doi: 10.3109/10409238.2012.758083

Tzin, V., and Galili, G. (2010). New insights into the shikimate and aromatic amino acids biosynthesis pathways in plants. Mol. Plant 3, 956-972. doi: 10.1093/mp/ ssq048

van de Loo, F. J., Broun, P., Turner, S., and Somerville, C. (1995). An oleate 12hydroxylase from Ricinus communis L. is a fatty acyl desaturase homolog. Proc. Natl. Acad. Sci. U.S.A. 92, 6743-6747. doi: 10.1073/pnas.92.15.6743

Vogt, T. (2010). Phenylpropanoid biosynthesis. Mol. Plant 3, 2-20. doi: 10.1093/ $\mathrm{mp} / \mathrm{ssp} 106$

Vranová, E., Coman, D., and Gruissem, W. (2013). Network analysis of the MVA and MEP pathways for isoprenoid synthesis. Annu. Rev. Plant Biol. 64, 665-700. doi: 10.1146/annurev-arplant-050312-120116

Wang, M., Toda, K., and Maeda, H. A. (2016). Biochemical properties and subcellular localization of tyrosine aminotransferases in Arabidopsis thaliana. Phytochemistry 132, 16-25. doi: 10.1016/j.phytochem.2016.09.007

Weng, J.-K. (2014). The evolutionary paths towards complexity: a metabolic perspective. New Phytol. 201, 1141-1149. doi: 10.1111/nph.12416

Weng, J.-K., Philippe, R. N., and Noel, J. P. (2012). The rise of chemodiversity in plants. Science 336, 1667-1670. doi: 10.1126/science.1217411

Wichers, H. J., Visser, J. F., Huizing, H. J., and Pras, N. (1993). Occurrence of L-DOPA and dopamine in plants and cell cultures of Mucuna pruriens and effects of 2,4-d and $\mathrm{NaCl}$ on these compounds. Plant Cell Tiss. Organ. Cult. 33, 259-264. doi: 10.1007/BF02319010

Williams, B. P., Aubry, S., and Hibberd, J. M. (2012). Molecular evolution of genes recruited into $\mathrm{C}_{4}$ photosynthesis. Trends Plant Sci. 17, 213-220. doi: 10.1016/j.tplants.2012.01.008

Yoo, H., Widhalm, J. R., Qian, Y., Maeda, H., Cooper, B. R., Jannasch, A. S., et al. (2013). An alternative pathway contributes to phenylalanine biosynthesis in plants via a cytosolic tyrosine:phenylpyruvate aminotransferase. Nat. Commun. 4:2833. doi: $10.1038 /$ ncomms 3833

Conflict of Interest Statement: The author declares that the research was conducted in the absence of any commercial or financial relationships that could be construed as a potential conflict of interest.

Copyright $\odot 2019$ Maeda. This is an open-access article distributed under the terms of the Creative Commons Attribution License (CC BY). The use, distribution or reproduction in other forums is permitted, provided the original author(s) and the copyright owner(s) are credited and that the original publication in this journal is cited, in accordance with accepted academic practice. No use, distribution or reproduction is permitted which does not comply with these terms. 